\title{
Finishing of helical gears using abrasive flow finishing
}

\author{
Dipti Sharma*, Kamal.k.kar and J.Ramkumar \\ Department of Materials ScienceProgramme and Mechanical Engineering, Indian Institute of \\ Technology Kanpur, Kanpur -208016, India \\ *Email: diptis@iitk.ac.in
}

Gear is a rotating part of machine having teeth that meshes with one another to transmit torque. They are used to vary speeds, change torque and power source direction. Gears are preferred over other drive mechanisms because of their constant velocity ratio. Gears are widely used in day to day life. Their applications are in automobiles, marine drives, agricultural equipment, conveyor systems, power stations, turbines, etc. Gear tooth surface generating process is not geometrically accurate and smooth. These inaccuracies and poor surface finish causes premature failure of gears. This results in reduction of power transmitting capacity, produces noise, excessive wear, backlash, pitting, scoring and surface cracks. To overcome these failures of gear tooth, finishing is required. Apart from surface finishing processes like gear shaving, roll finishing, gear grinding, lapping and honing, a non-conventional machining method is abrasive flow finishing (AFF) is used.

Even the most inaccessible areas can be finished by AFF which can process multiple holes, slots or edges in a single operation [1]. As manual machining is challenging so automated AFFs are used to significantly reduce the labor cost.

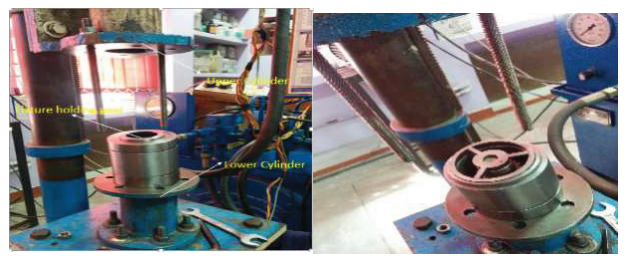

Figure 1: Arrangement of AFF, fixture and workpiece (left), Top view and zoomed view (right)

AFF consists of three parts- machining, tooling and medium. Among its several variants, two-way flow AFF is found to have the most suitable machining set up to carry out the finishing process. In present study, two-way flow AFF has upper and lower cylinders in which piston reciprocates along with medium. Tooling has a fixture which holds helical gear having helix angle $30^{\circ}$ and $45^{\circ}$ and provides a pathway for medium to flow over gear only. Medium is composed of abrasives, a base polymer carrier and additives in the form of plasticizer and softeners.

Mixing of styrene butadiene rubber (SBR) and abrasive particles is carried out using two rolls mill, which ensures uniform dispersion. Complex shapes are easy to finish due to viscoelastic nature of medium used in finishing. Medium viscosity can be changed by varying the amount of additive in it [2]. As the plasticizer content increases, viscosity of medium reduces because of diffusion of plasticizer (low molecular weight molecule) into polymer molecular chains, thereby reducing intermolecular forces between them. Higher viscosity reduces the flowability of medium whereas low viscosity does not give the desired roughness value. Therefore, it is important to have an optimum viscosity of medium.So quantitative measure of viscosity and its variation was found using parallel plate rheometer.

In the present work, an experimental investigation of process parameterslike viscosity, effects of percentage of various components in medium, number of cycles and helix angle of gears have been studied on Ra value of gear surface. The experimental results has shown a 35\% improvement in Ra value of helical gears having helix angle $30^{\circ}$ and $45^{\circ}$, after operating for 900 cycles.

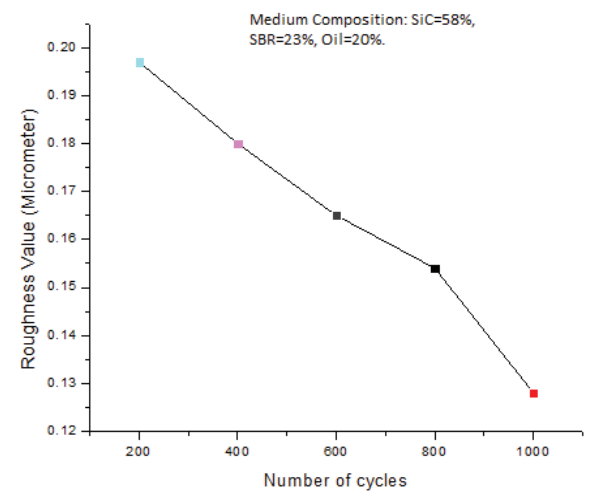

Figure 2: Graph showing variation of surface roughness value versus number of cycles

Figure 2 above shows variation in surface roughness values with number of cycles. This graph shows improvement in surface finish with increase in number of finishing operations, due to shearing off of the peaks on helical gears.

\section{References}

1. Rhoades, L. Journal of Materials Processing Technology, 1991. 28(1): p. 107-116.

2. Kar, K.K., et al., Journal of Manufacturing Science and Engineering, 2009. 131(1): p. 011009. 